\title{
A Build Up of Seizure Prediction and Detection Software: A Review Article
}

\author{
Hadriche $\mathrm{A}^{1,2}$ and J mail $\mathrm{N}^{2,3,4 *}$ \\ ${ }^{1}$ REGIM Lab, ENIS, Sfax University, Sfax, Tunisia \\ ${ }^{2}$ Digital Research Center of Sfax, Sfax, Tunisia \\ 3INS, UMR 1106, Aix Marseille University, Marseille, \\ France \\ ${ }^{4}$ MIRACL Lab, Sfax University, Sfax, Tunisia \\ *Corresponding author: Nawel J mail, INS, UMR \\ 1106, Aix Marseille University, Marseille, France; \\ MIRACL Lab, Sfax University, Sfax, Tunisia ; Digital \\ Research Center of Sfax, Sfax, Tunisia
}

Received: April 16, 2021; Accepted: May 06, 2021; Published: May 13, 2021

\begin{abstract}
Introduction: Neurological diseases are much often due to our stressed daily life, and epilepsy is considered as a second cause of hospitalization in neurological illness. It is about $30 \%$ of epileptic cases where medicine would not stop or control seizure; hence, a surgical intervention is required to delineate abnormal hyperexcitable cortical tissue. Defining these epileptogenic zones is a challenge that require physiological and anatomical acquisition.
\end{abstract}

Discussion: Clinicians, researcher and engineer researcher are multiplying advanced techniques in order to exploit these acquisitions for a better diagnosis. Several software are used to enhance epilepsy diagnosis. Here we proposed a software that rely on space-time evolution of inter- ictal gamma oscillations.

Conclusion: Our proposed software would predict a build up of seizure during monitoring of stereo-electroencephalographic SEEG recording. It allows also detection of seizure during analysis and diagnosis of SEEG. This software would assist neurologist in recognition of seizure and in defining epileptogenic zone EZ.

Keywords: Pharmaco-ressistant epilepsy; SEEG; Seizure build up; Prediction; Detection

\section{Introduction}

In $20 \%$ of cases, epilepsy is misdiagnosed for several reasons: non-specialized health personnel, and non-efficient analysis of physiological and anatomical registration. Moreover, this neurological disease requires a diagnostic wandering that dragged on average 5 years. About $70 \%$ of epileptic subjects could be seizure free if they had an appropriate treatment. Hence, the trend actually is oriented to develop more decision-support tools and improve diagnosis for epilepsy. 30\% of epileptic cases are pharmaco ressistant, requiring invasive recording and surgical intervention to delineate epileptogenic zones responsible of excessive discharges and build up seizure. For a long time epileptic spikes are considered as the best biomarker that should be preprocessing to explore physiological registration and describing EZ [1]. Furthermore, scientists proved that oscillations from gamma to ripple to high frequency oscillation HFO could also be surveyed as an important biomarker to detect hyperexcitability tissue [2].

In this review we present a software to assist neurological clinician during pharmaco resistant epilepsy diagnosis; this software rely on detecting interictal gamma oscillations in first step, then defining their spatio temporal map to predict a build up of seizure during monitoring of SEEG signal $[3,4]$.

This software could also detect seizure through analysis of interictal gamma oscillations. Detecting timing and spatial extent are highly important indices for clinician during epilepsy diagnosis.

\section{Discussion}

Software to assist clinician during epilepsy diagnosis are very widespread, with basic principles that varies between registrations, and analyzed biomarkers $[5,6]$.

There is Software capable to inspect both invasive and non invasive registration, other for anatomical images. For epileptic biomarker, spiky events present the highest analyzed biomarker in epilepsy for decades [7], but recently studies are oriented to oscillatory events from low gamma to very high gamma HFO [5]. Studying epileptic oscillatory events depends straightly on type of used registration, since HFO are much more seen in SEEG than in non invasive technique. MEG did also depict more gamma oscillation in some cases than EEG. Hence, it seems promising to combine the study of different modalities of registration in order to detect efficient biomarker. In fact, studying and analyzing these biomarkers is coherent with the designation of region with excessive discharges and build up of a seizure [3].

In this review, we present a software capable of detecting pure interictal gamma oscillations non- contaminated by epileptic spikes using "despiking" of SEEG signal, in order to predict a build up of seizure and detect a seizure among SEEG recordings [3,9].

This software is also capable to assist clinician in defining EZ since it depicts the space extent of cortical region with excessive discharges.

Despiking is an advanced technique that relies on creating a spiky basis than projecting investigated signal on spiky model [3], resulting signal is pure interictal gamma oscillations [8]. In a second step, a spatio temporal analysis is applied to elucidate time of incoming seizure and responsible EZ.

This advanced preprocessing technique of predicting and 


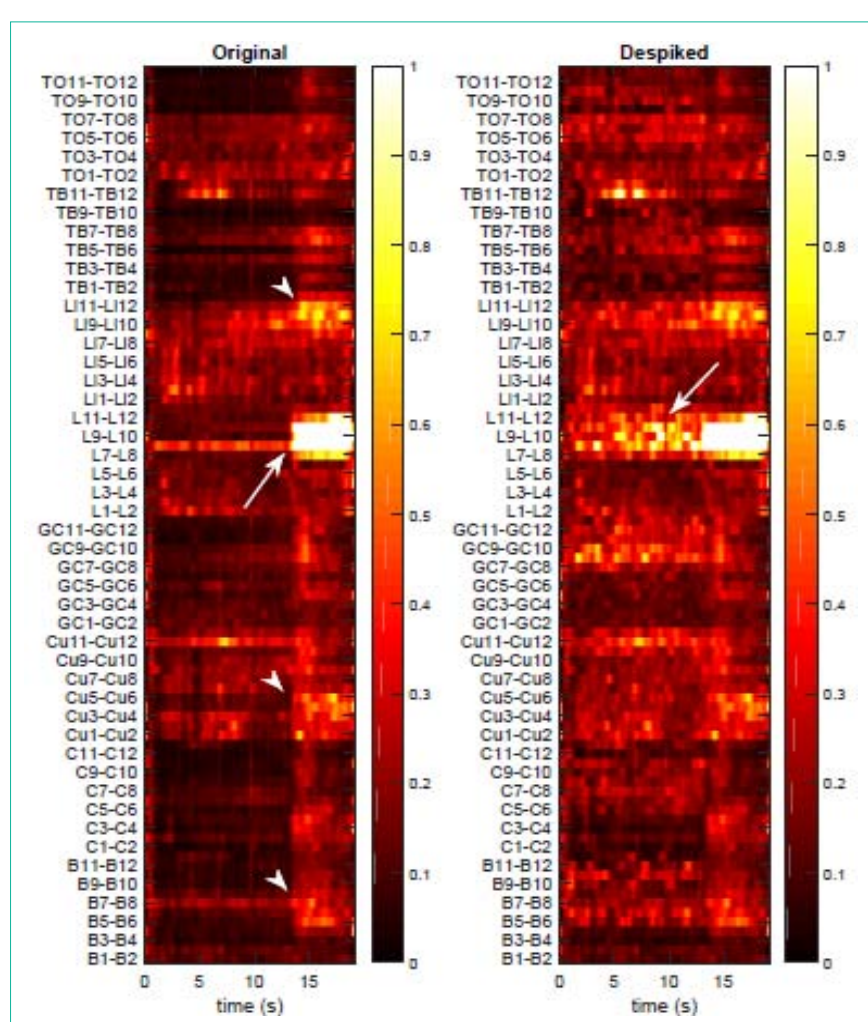

Figure1: Spatio-temporal maps of SEEG signals (channels $x$ time), (a) Original SEEG with no clear pattern in preictal period (between 0 and $14 \mathrm{~s}$ ). Seizure is seen in several regions (arrows), with maximum energy ratio in channels L7 and L8 to L11 and L12 (arrow with tail). (b) Despiked signals, a clear 'build-up' of oscillatory activity and seizure are clear in channels L8 and L9 to L10 and L11 (arrow with tail) [10]. This software was capable to predict the incoming seizure by providing the exact start timing $13 \mathrm{~s}$ and exact spatial extent L8 to L11.

recognition of a seizure was validated on both simulated (inspired from real SEEG signal) and SEEG signal $[9,10]$.

SEEG recording investigated in this review is a presurgical stereo-electroencephalography data, diagnosed as a drug-resistant symptomatic focal cortical dysplasia FCD in the right occipitotemporal junction focal epilepsy. SEEG was recorded at the Clinical Neurophysiology Department of the Timone hospital in Marseille, using eight multi-contact depth electrodes with $0.8 \mathrm{~mm}$ diameter, 10 to 15 contacts, each $2 \mathrm{~mm}$ long with an inter-contact distance of $1.5 \mathrm{~mm}$. These electrodes are implemented in the right hemisphere, according to Talairach's stereotaxic method [11,12]. Deltamed System record SEEG signal with $256 \mathrm{~Hz}$ sampling frequency, depicting clear and abundant preictal patterns with regular spiking and visible epileptic oscillations. Patients signed informed consent, and the study was approved by the Institutional Review board (IRB00003888) of INSERM (IORG0003254, FWA00005831).

We investigate our SEEG signal using our proposed software to predict a build up of a seizure using non contaminated interictal gamma oscillations. In figure one, we depict prediction of a build up seizure (time and space), after a despiking step followed by a spatio temporal analysis.

\section{Conclusion}

Pharmaco resistant epilepsy, is a traumatic disease, unforeseeable seizure with a lot of suffering, it is in this context that, diagnostic tools should be improved and boosted in order to recognize and predict occurrence of a build up of a seizure. This proposed software will have positive impacts on the quality of clinicians' diagnosis [13] as well as on well-being of epileptic patients. Our proposed software, in this review, ensures a prediction and detection of seizures occurrence through SEEG recordings in order to improve the diagnosis efficiency and physical as well as psychological state of pharmaco-resistant patient.

\section{References}

1. Wendling F, Hernandez A, Bellanger J-J, Chauvel P, Bartolomei F. Interictal to ictal transition in human temporal lobe epilepsy: insights from a computational model of intracerebral EEG. J Clin Neurophysiol. 2005; 22 343-356.

2. Urrestarazu E, Jirsch JD, LeVan P, Hall J, Gotman J. High-frequency intracerebral EEG activity $(100-500 \mathrm{~Hz})$ following interictal spikes. Epilepsia. 2006; 47: 1465-1476.

3. Jmail N, Gavaret M, Bartolomei F, Benar C-G. Despikifying SEEG signals using a temporal basis set. In Proceedings of the $201515^{\text {th }}$ International Conference on Intelligent Systems Design and Applications (ISDA). IEEE. 2015; 580-584.

4. Jmail N, Jarray R, Hadrich A, Frikha T, Benar C. Separation between spikes and oscillation by stationary wavelet transform implemented on an embedded architecture. J Neurol Sci. 2017; 381.

5. Jmail N, Gavaret M, Bartolomei F, Chauvel P, Badier JM, Bénar CG. Comparison of Brain Networks During Interictal Oscillations and Spikes on Magnetoencephalography and Intracerebral EEG. Brain Topogr. 2016; 29.

6. Frikha T, Hadriche A, Khemakhem R, Jmail N, Abid M. Adaptive architecture for medical application case study: evoked Potential detection using matching poursuit consensus. International Conference on Intelligent Systems Design and Applications. 2015; 575-579.

7. Hadriche A, Jmail N, Kachouri A, Ghariani H. The detection of Evoked Potential with variable latency and multiple trial using Consensus matching pursuit. International Conference on Advanced Technologies for Signal and and Image Processing. 2014; 22-31.

8. Hadriche A, Jmail N, Elleuch R, Pezard, L. Different Methods for Partitioning the Phase Space of a Dynamic System. Int J Comput Appl. 2014; 93: 1-5.

9. Jmail N, Zaghdoud M, Hadriche A, Frikha T, Ben Amar C, Bénar C. Integration of stationary wavelet transform on a dynamic partial reconfiguration for recognition of pre-ictal gamma oscillations. Heliyon. 2018.

10. Abdennour N, Hadriche A, Frikha T, Jmail N. Extraction and localization of non-contaminated alpha and gamma oscillations from EEG signal using finite impulse response, stationary wavelet transform, and custom FIR, International Conference on Artificial Neural Networks. 2018; 511-520.

11. Jmail N, Gavaret M, Bartolomei F, Bénar C-G. Despiking SEEG signals reveals dynamics of gamma band preictal activity. Physiological Measurement. 2017; 38: $42-56$.

12. Talairach J, Bancaud J. Tereotaxic approach to epilepsy. Methodology of anatomo-functional stereotaxic investigations. Prog Neurol Surg. 1973; 5 : 297-354.

13. Hadriche A, Jmail N, Blanc JL, Pezard L. Using centrality measures to extract core pattern of brain dynamics during the resting state. Computer methods and programs in biomedicine. 2019; 179: 104985. 